\title{
Targetoid erythema surrounding multiple seborrheic keratoses induced by chemotherapy with gemcitabine
}

\author{
Chiara Retrosi ${ }^{1}$, Roberta Vezzoni ${ }^{1}$, Claudio Conforti ${ }^{1} \bowtie$, Paola Corneli ${ }^{1}$, Giovanni Magaton-Rizzi ${ }^{1}$, Iris Zalaudek ${ }^{1}$, Nicola Di \\ Meo $^{1}$
}

\begin{abstract}
The cutaneous adverse effects of gemcitabine include allergic skin rash frequently associated with pruritus, alopecia, sweating, dermatitis with boils, and ulcerations. We report the case of a patient that developed inflammation of seborrheic keratoses after gemcitabine treatment.
\end{abstract}

Keywords: gemcitabine, seborrheic keratoses, drug adverse reaction

Received: 8 July 2019| Returned for modification: 22 August 2019 | Accepted: 13 November 2019

\section{Introduction}

Seborrheic keratosis is the most common benign skin tumor of middle-aged and elderly adults. Seborrheic verrucae are benign skin lesions, usually brownish, that are mainly located on the chest but can develop in any skin region (1).

Chemotherapy of internal neoplasms or eczemas may lead to the onset of seborrheic keratosis or promote their inflammation (2). The literature includes numerous adverse effects of chemotherapy drugs on the skin, and in particular several cases of nucleoside analogues (such as gemcitabine) known to cause inflammation of seborrheic keratosis (3-6).

The authors report a case of inflammation of seborrheic keratosis after administration of gemcitabine.

\section{Case report}

A 78-year-old woman with right lung adenocarcinoma (Stage IV), diagnosed in April 2018, came to our attention for a newly emerging erythematous purple-colored skin rash spread all over the back. Clinically numerous preexisting seborrheic keratoses were clearly evident on the patient's back, and the rash spared the perilesional skin around these. She denied having taken new drugs in the last month with the exception of chemotherapy; in fact, she received six cycles of chemotherapy with gemcitabine and cisplatin from September until October 2018, and subsequently she developed intensive itching, erythema, edema, and scaling in multiple preexisting seborrheic keratoses.

On physical examination, an erythematous rash was observed on the back, especially on some seborrheic keratoses on which targetoid lesions with saving of the skin surrounding keratosis were observed (Fig. 1a). The patient did not have a fever, but only asthenia and generalized itching.

A dermoscopic examination (20×, Dermatoscope DermLite $3 \mathrm{Gen}$ ) was carried out and showed dotted and linear vessels and a purplish-erythematous background surrounding seborrheic keratosis. The numerous seborrheic keratoses were dermoscopically confirmed based on evidence of classic criteria: milia-like cysts, comedo-like openings, gyri and sulci, and network-like structures (Fig. 1b).
In our case we did not consider it appropriate to resort to a histological examination for diagnosis because today dermoscopy is a diagnostic tool with very high sensitivity and specificity for most skin lesions. Therefore, evaluating the history, as well as the clinical and dermoscopic examination of the skin manifestation, we considered it unnecessary to perform a biopsy.

Diagnosis of seborrheic keratosis with targetoid inflammation related to chemotherapy was made, and a cycle of methylprednisolone $40 \mathrm{mg}$ to scale for 10 days was started, resulting in complete resolution of the clinical cutaneous manifestations.

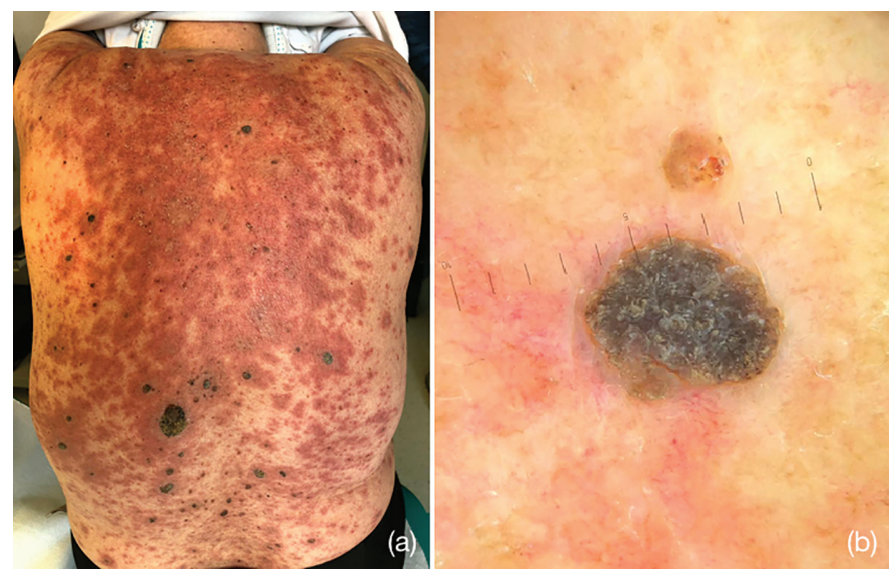

Figure $1 \mid$ a) Clinical image of the back. Erythematous rash with targetoid lesions with saving of the skin surrounding seborrheic keratosis. b) Dermoscopy (polarized $\times 20$ ) exhibits an erythematous purplish background around the keratosis with dotted and linear vessels. The diagnosis of seborrheic keratosis was based on classic and well-described criteria of seborrheic keratosis such as milia-like cysts, comedo-like openings, gyri and sulci, and network-like structures.

\section{Discussion}

Many adverse skin events to chemotherapy drugs are described in the literature and, in particular, nucleoside analog drugs such as gemcitabine are known to cause inflammation of seborrheic keratoses. Based on the scientific evidence and the absence in the literature of a correlation between cisplatin and targetoid reactions, we considered the skin reaction described to have most likely been caused by gemcitabine.

Inflammation of seborrheic keratoses is a rarely described cutaneous reaction of chemotherapy (7), and in the literature there 
is only one previous case report mentioning this skin reaction (8), in which a patient with pancreatic cancer received chemotherapy with gemcitabine and developed an inflammatory reaction on preexisting seborrheic keratoses.

Cases of inflammation of seborrheic keratoses induced by other chemotherapy drugs such as cytarabine, 5-fluorouracil, docetaxel, vincristine, and doxorubicin are described (3-6), but as reported in the literature these are predominantly nucleoside analogues.

The majority of systemic adverse effects of chemotherapy are most often predictable, such as nausea, vomiting, diarrhea, decreased white blood cells, decreased immune defenses, and anemia, as well as common side effects on the skin and annexes: alopecia, erosive stomatitis, or aphthous ulcers. There are also other side effects such as inflammation of benign skin tumors, which is more rarely observed and less commonly diagnosed than side effects of chemotherapy (9).

\section{References}

1. Ranasinghe GC, Friedman AJ. Managing seborrheic keratoses: evolving strategies for optimizing patient outcomes. J Drugs Dermatol. 2017;16:1064-8.

2. Fitzpatrick JE, Yokel BE, Hood AF. [Complicações mucocutâneas da terapia antineoplásica]. In: Freedberg MI, Eisen AZ, Wolff K, Austen KF, Goldsmith LA, Katz SI, Fitzpatrick TB, editors. Fitzpatrick tratado de dermatologia. 5 ed. Rio de Janeiro: Revinter; 2005. p. 1642-53. Portuguese.

3. Patton T, Zirwas M, Nieland-Fisher N, Jukic D. Inflammation of seborrheic keratosis caused by cytarabine: a pseudo sign of Leser-Trelat. J Drugs Dermatol. 2004;3:565-6.

4. Chu CY, Yang CH, Chiu HC. Inflammation of seborrheic keratoses due to docetaxel treatment. Acta Derm Venereol. 2001;81:316-7.

5. Kechijian P, Sadick NS, Mariglio J, Schulman P. Cytarabine-induced inflammation in seborrheic keratoses of Leser-Trelat. Ann Intern Med. 1979;91:868-9.
The mechanism of action causing this side effect is not known; it has been hypothesized that cytotoxic damage directed against keratinocytes may be at work, whereas in other studies an infiltrate of lymphocytes into the dermis appears to be the cause of these skin lesions (7).

It is possible that chemotherapy treatment may involve changes in dosage or the method of administration (e.g., a cycle may be postponed for a week) precisely because of the toxic effects.

\section{Conclusions}

In the case we describe, it was not necessary to interrupt the chemotherapy because, although the clinical presentation was very extensive, it responded well to systemic corticosteroid therapy. For the dermatologist it is essential to recognize at an early stage the adverse effects caused by chemotherapy agents for early drug treatment without the need to stop the oncological therapy.

6. Williams JV, Helm KF, Long D. Chemotherapy-induced inflammation in seborrheic keratosis mimicking disseminated herpes zoster. J Am Acad Dermatol. 1999;40:643-4.

7. Susser WS, Whitaker-Worth DL, Grant-Kels JM. Mucocutaneous reactions to chemotherapy. J Am Acad Dermatol. 1990;40:367-98.

8. Siqueira CR, Miot HA. Inflammation of multiple seborrheic keratoses induced by chemotherapy with gemcitabine. An Bras Dermatol. 2009;84:410-3.

9. Grob JJ, Rava MC, Gouvernet J, Fuentes P, Piana L, Gamerre M, et al. The relation between seborrheic keratoses and malignant solid tumors: a case-control study. Acta Derm Venereol. 1991;71:166-9. 